\title{
Shiga Toxin Producing Escherichia coli Infections and Associated Haemolytic Uraemic Syndrome in New Zealand Children: Twenty Three Years of Epidemiology and Clinical Observations
}

\author{
William Wong, MB, ChB, FRACP* \\ Department of Paediatric Nephrology, Starship Children's Hospital, New Zealand
}

*Corresponding author: William Wong, MB, ChB, FRACP, Department of Paediatric Nephrology, Starship Children's Hospital, Auckland District Health Board, Private Bag 92024, Auckland, New Zealand, Tel: +64-9-3078900, Fax: +64-9375-4371

\begin{abstract}
Background: The epidemiology of childhood haemolytic uraemic syndrome in New Zealand was on active surveillance from January 1, 1998 to December 31, 2020. The aim of this study is to describe the demographic, epidemiological and some clinical features of childhood Shiga toxin producing $E$. coli infections (STEC) and its association with diarrhoea associated haemolytic uremic syndrome (D+HUS) over past 23 years.

Methods: The New Zealand Paediatric Surveillance Unit (NZPSU) sent out a monthly report card to all practising paediatricians with conditions under active surveillance. Those paediatricians who had cared for a child aged 0 to 15 years of age with D+HUS in the past month were requested to report their patient to the NZPSU. The reporting clinicians were then contacted by the principal investigator who sent out a questionnaire to the clinician requesting patient clinical and laboratory information. The group was divided into 2 cohorts; A: 1998-2008 and B: 2009-2020 to examine trends in STEC infections and childhood D+HUS.

Results: Two hundred and twenty six children aged 3 months up to 15 years (median 2.8 years (IQR 1.7-4.9) were diagnosed with D+HUS. E. coli O157: $\mathrm{H} 7$ was identified in $130(57.1 \%)$ and 8 were due to serotypes $026 \mathrm{H} 11$ and 0113. Over the 23 years, the mean annual incidence in children under age 5 between cohort $A$ and cohort $B$ was not statistically significant (2.2 per 100,000 vs. 2.85 per 100,000 . The mean annual incidence was 1.2 per 100,000 in all children under age 15 for the whole study period. Disease severity as measured by the requirement for acute dialysis had not changed and was required in $128 / 226(56.2 \%)$ of
\end{abstract}

children for a median of 9 days (range 1-38). The overall mortality was $3 / 226(1.3 \%)$, which was due exclusively to severe cerebral injury.

Conclusion: There has been an increase in STEC infections in childhood over the past 23 years, in part due improved methods of detection, however, the incidence children developing D+HUS has remained stable over the study period. E. coli serotypes other than $0157: \mathrm{H} 7$ have increased in prevalence over the past 6 years due to methodological changes in diagnostic laboratories, but with only a small number of infected children developing D+HUS. The mortality rate over the study period was low, consistent with other reported series.

\section{Keywords}

Shiga toxin producing E. coli, Haemolytic uraemic syndrome, Children, Demographics, Epidemiology

\begin{abstract}
Abbreviations
AKI: Acute Kidney Injury; Cl: Confidence Interval; D+HUS: Diarrhoea Associated Haemolytic Uraemic Syndrome; $E$ coli: Escherichia coli; ESR: Environmental Science and Research; HUS: Haemolytic Uraemic Syndrome; g/L: grams/litre; Hb: Haemoglobin; IQR: Interquartile Range; NZPSU: New Zealand Paediatric Surveillance Unit; OR: Odds Ratio; stx: Shigatoxin; SD: Standard Deviation
\end{abstract}

\section{Introduction}

Diarrhoea associated haemolytic uraemic syndrome (D+HUS) secondary to gastrointestinal infection is the most common cause of haemolytic uraemic syndrome 
in children. It is the result of ingestion food or water that has been contaminated with Shigatoxin-producing Escherichia coli (STEC). Haemolytic uraemic syndrome (HUS) consists of microangiopathic haemolytic anaemia, thrombocytopenia and acute kidney injury. Thrombotic microangiopathy underlies this disorder, and is a consequence of toxin mediated vascular endothelial injury in kidney, brain and other organs, leading to platelet aggregation and consumption, red cell fragmentation causing micro-thrombi which result in ischaemic injury to vulnerable organs. Treatment of the end organ injury consists of supportive measures such as careful fluid management, dialysis, red cell transfusions and the management of extrarenal complications such as seizures [1-5].

STEC-associated HUS (STEC-HUS) occurs worldwide with E. coli 0157:H7 being the most commonly recovered serotype [6-8]. Some of the predominance of E. coli $0157: \mathrm{H} 7$ is attributable to the ease with which this organism is distinguished on agar plates by its sorbitol nonfermenting phenotype [9]. Clinical microbiology laboratory routinely tests all stool cultures for $E$. coli 0157, using its inability to ferment sorbitol on sorbitolMacConkey (SMAC) agar. E. coli 0157 infections may be missed on SMAC because some strains ferment sorbitol and cannot be differentiated from normal intestinal flora. Chromogenic agar was introduced to some laboratories in other countries to improve the detection of $E$. coli $\mathrm{O} 157$ through its ability to produce a distinctive colour change due to chromogenic substances in the medium [10]. This form of culture medium had been in limited use in New Zealand laboratories. However, the advent of nucleic acid amplification technology and toxin enzyme immunoassays has demonstrated that non-0157:H7 STEC serotypes can also cause HUS [1114].

STEC-HUS is a common cause of acute kidney injury (AKI) in children requiring acute kidney replacement therapy in developed countries [15-17]. Over the past two decades, our own experience has shown that a large proportion of children with acute kidney injury who have required acute dialysis were due to D+HUS [18]. Some these patients have required prolonged hospitalisation, multiple operating room procedures, blood transfusions and other associated procedures.

In 1997 the New Zealand Paediatric Surveillance Unit (NZPSU) in association with the University of Otago, Dunedin, instituted a surveillance system to obtain epidemiological data on rare diseases, such as HUS, which is uncommon despite its role as a common cause of severe AKI in otherwise healthy children. Accordingly, HUS was listed as a reportable condition for surveillance beginning January $1^{\text {st }} 1998$ until $31^{\text {st }}$ December 2020 [19]. We describe the epidemiology and clinical features of D+HUS collected through the NZPSU over the past twenty-three years.

\section{Methods}

Surveillance was conducted via a monthly report card sent to all practising paediatricians in New Zealand requesting information on any cases of HUS in which they have been involved in the previous month. Respondents who return an affirmative response were contacted by the principal investigator who then sent out a detailed questionnaire requesting demographic clinical and laboratory information. Positive STEC detections were notified to the regional public health authorities as suspected STEC infections invoked a mandatory report to public health authorities to a confirmed STEC infection and subsequent source identification. Response rates of paediatricians notifying the NZPSU have been excellent, varying from $94 \%$ between $2005-2009$, 91.5\% 20102014 and 90\% from 2015-2020. Demographic, clinical and laboratory data were collated from completed questionnaires from paediatricians who had reported clinical care of a child with HUS (Supplementary File (Questionnaire)). Clinical features recorded included seizures, presence of hypertension, presence of anuria and its duration, requirement for and duration of acute dialysis. The prodrome period was defined as the time between onset of diarrhoea and clinical diagnosis of HUS, and the identification of a plausible bacterial agent was also included. Complications of HUS such as death, persistent hypertension, chronic kidney disease, cardiac complications, diabetes and pancreatitis were documented. The questionnaire was first developed in 1998 and has remained unchanged until 2008, when a question concerning any recent visit to a farm within two weeks of the presenting diarrhoeal illness was added.

The case definition of D+HUS was made on history of vomiting and or diarrhoea in any child less than 15 years of age with microangiopathic haemolytic anaemia (Haemoglobin < $100 \mathrm{~g} / \mathrm{L}$ ) with microscopic evidence of fragmented red blood cells, thrombocytopenia (Platelet count $<150,000 \times 10^{9}$ ) and acute kidney injury (oliguria or anuria with elevated serum urea and creatinine for age and gender of child [19]. All children with other causes of HUS were excluded. Historically, STEC were detected by standard bacterial culture methods (plating stool on sorbitol MacConkey agar or other chromogenic agar to detect E. coli $0157: \mathrm{H} 7)$ and serological identification of the STEC, as confirmed by the Institute of Environmental Science and Research, Kenepuru, Porirua, New Zealand [20]. Chromogenic agar, CHROMagar STEC was introduced in 2011 to our hospital microbiology service but did not gain widespread usage through the rest of the country (Fort Richard Laboratories, New Zealand, www.fortrichard. com) Molecular testing for genes encoding Shiga-toxins was introduced in 2015 and phased into various hospital and community laboratories around the country over six years. This is a commercially available platform for the detection of a range of gastrointestinal infections [21]. 

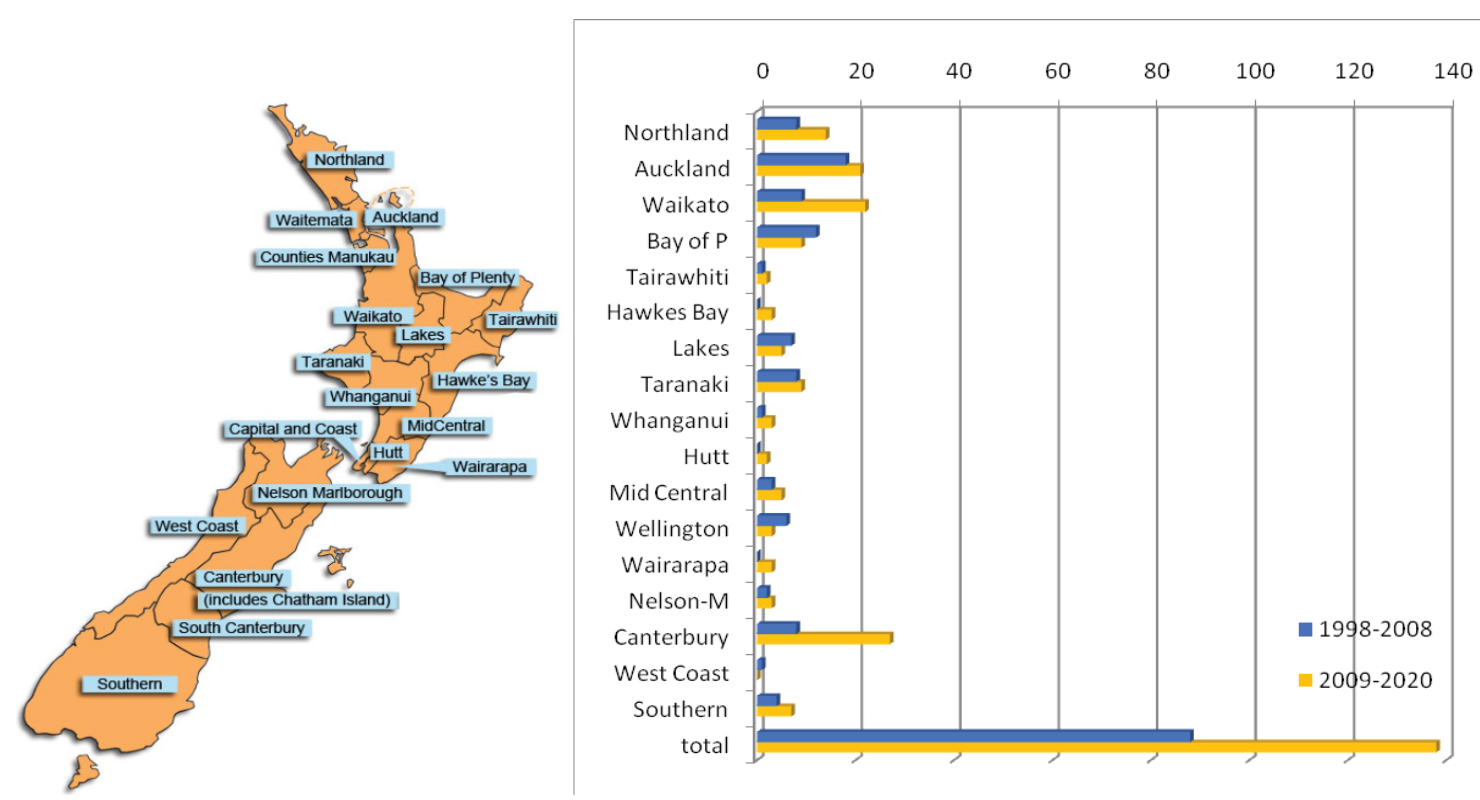

Figure 1: Haemolytic uremic syndrome children by district health board region, 1998-2007 vs. 2008-2017.

Bay of P: Bay of Plenty; Nelson M: Nelson Marlborough; Capital Coast Wellington Use of the District Health Board Regions map was approved by the Ministry of Health, New Zealand.5.05.2020. https://www.health.govt.nz/new-zealand-healthsystem/key-health-sector-organisations-and-people/district-health-boards/location-boundaries-map

Cases were assigned to specific districts health areas based on the domicile of the patient where the diarrhoeal illness had developed. The Auckland region has three health districts and the number of cases was combined into one value. The study population was divided into two cohorts; cohort A, January 1, 1998 to December 31, 2008 and cohort B, January 1, 2009 to December 31, 2020 to show trends in the epidemiology of STEC infections and HUS over the duration of the study. Census data obtained from Statistics New Zealand from 2001 through to 2018 were used to calculate regional incidence rates [https://www.stats.govt.nz].

Statistical analyses were performed using Graphpad Instat version 3.1 for Windows and Graphpad Prism 4.0. [22]. Continuous data are expressed as mean \pm standard error of the mean (SEM) and compared using Student t-test. Categorical variables were represented as percentages of the total in each group and were compared using Chi-square or Fisher exact tests as appropriate. All P-values are two tailed and significance was defined as a $p$ value $<0.05$.

The surveillance study was approved by the University of Otago Medical School Research Ethics committee and the research was undertaken in accordance to the guidelines of the Declaration of Helsinki.

\section{Results}

Two hundred and twenty-six children aged 3 months to 15 years were reported to the NZPSU with the diagnosis of D+HUS during the study period. There were 127 females with $74.8 \%$ of the whole group being less than age five. There were 88 patients in cohort $A$, (1998-2008) and 138 in B (2009-2020). There was no significant difference in the age of diagnosis between males and females or between the two time cohorts. The mean age of presentation was $3.8 \pm 0.2$ years (SEM); median 2.8 years (interquartile range 1.7-4.9). Sixteen children (7\%) under 12 months of age had diarrhoea associated HUS. One hundred and sixty-seven (73.4\%) were referred to the paediatric nephrology centre in Auckland for further care, which is the only facility in the country that offers acute dialysis or kidney replacement therapy to children.

\section{Geographical distribution}

All regions of New Zealand have recorded children with HUS with a trend for increasing case rate in the Canterbury and Waikato; 70\% (8 to 27 cases) and 59\% increase (9 to 22 cases) respectively, whereas other regions have recorded a reduction (Figure 1). Increase in the in the Canterbury and Waikato childhood population over the study period was $5 \%$ and $8.3 \%$ respectively. In contrast in Auckland, the most populous region in the country, the annual incidence had remained unchanged. There had a been more than 4-fold increase in reported STEC infections amongst children in the past 23 years, with a trend towards higher numbers over during period B (Figure 2 and Figure 3). There has been a recent reduction in absolute HUS cases in the Waikato and Canterbury (Figure 4).

\section{Bacteriology}

In the 226 children with D+HUS, 138 (61\%) cases had a Shiga toxin E. coli identified, of which 130 (94\%) was E. coli $\mathrm{O} 157: \mathrm{H7}$, and a further 8 children showing serotypes E. coli $\mathrm{O} 113$ and $\mathrm{O} 26 \mathrm{H} 11$. The distribution of stx1/stx2 positivity in New Zealand E. coli 0157:H7 


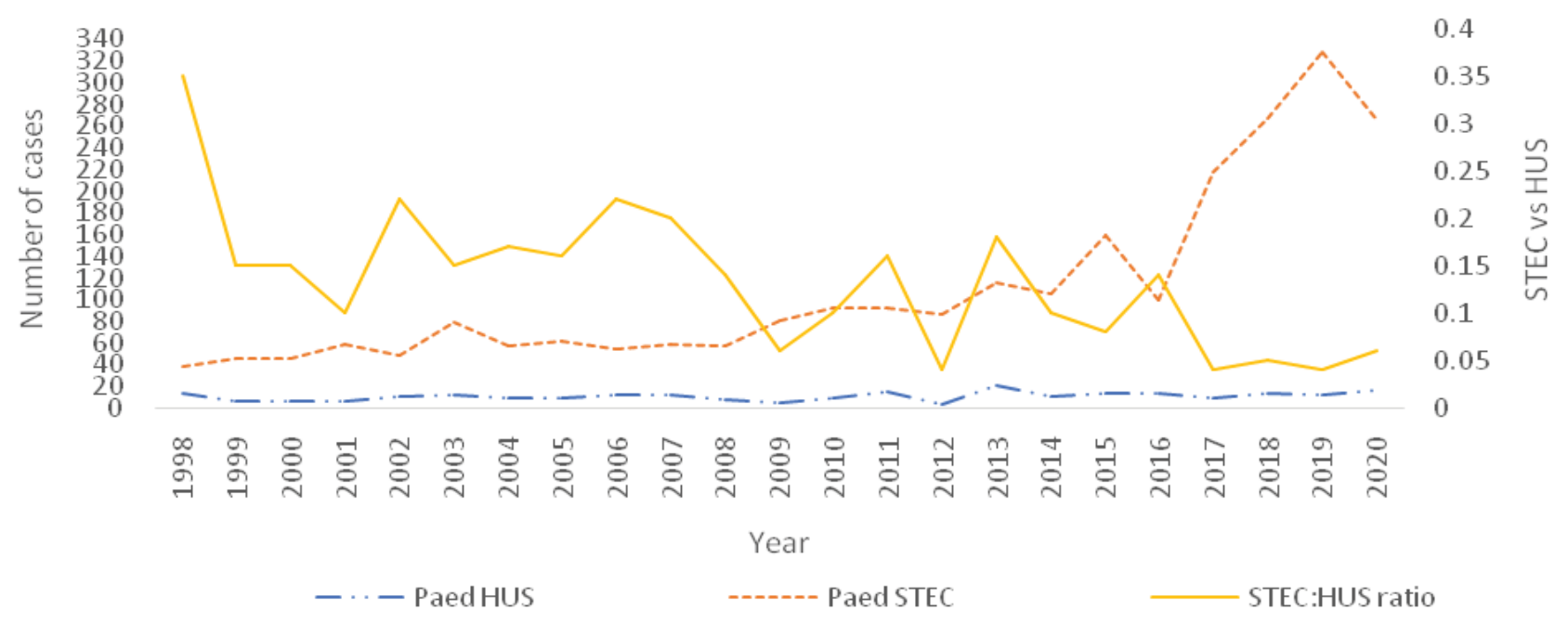

Figure 2: Number of children with STEC compared with HUS cases over 23 years.

A: Introduction of molecular testing for Shigatoxin; Paed: paediatric

Source: Institute of Environmental Scientific Research New Zealand- https://surv.esr.cri.nz/episurv/index.php (with permission).

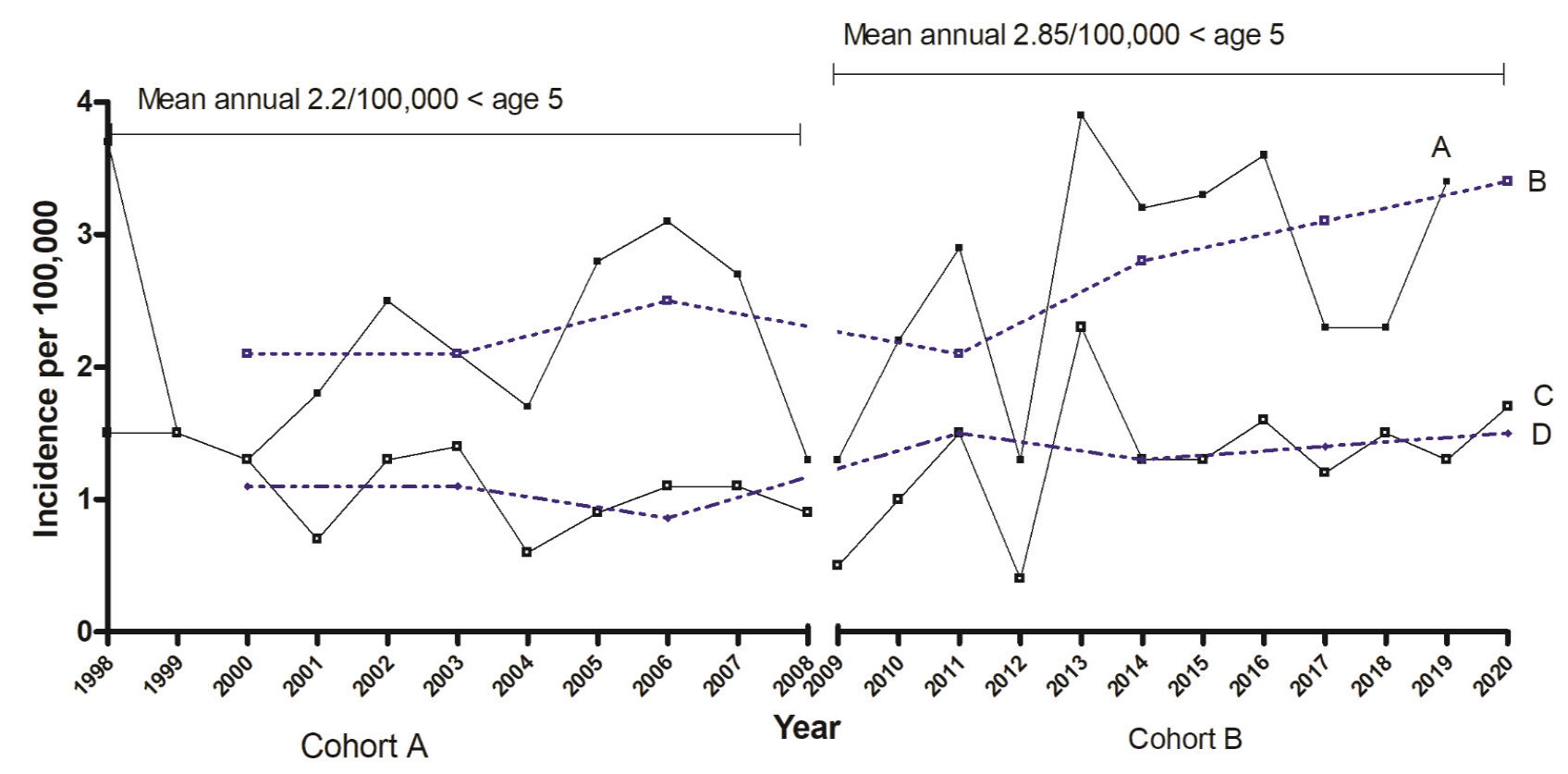

Figure 3: Annual and mean incidence in age groups $<5$ years and $<15$ years over study periods $1998-2008$ and 20092020.

Solid lines show annual incidence; broken lines: 3 yearly averaged incidence

Lines $A+B$ represent age group $<5$ years, lines $C+D$ represent age group 0-15 years.

isolates over the study period was stx1- stx2 $+(83 \%)$ and $\operatorname{stx} 1+\operatorname{stx} 2+(17 \%), 99 \%$ of New Zealand $E$. coli 0157:H7 express stx2 (personal communication). A potential source of infection was identified in 83/226 (36.7\%) children, of which $85.5 \%,(71 / 83)$ had a history of a farm contact within 2 weeks of diarrhoea onset. Five children developed HUS following ingestion of unpasteurised or raw milk. Three children with HUS had Campylobacter jejuni detected in their stools, but were negative for E. coli 0157:H7. A further 3 children had polymicrobial stool isolates containing Cryptosporidium and Aeromonas species.

\section{Duration of prodromal illness and bloody diarrhoea (Table 1)}

Two hundred twenty-two patients of the total cohort, (98.2\%) had the duration of diarrhoea onset to HUS diagnosis recorded with a median of 6 days (IQR 4-7) from the onset of diarrhoea to diagnosis of HUS. There was a small but significant improvement in the mean time to HUS diagnosis by one day between cohort $A$ and $B$. The history of bloody diarrhoea significantly increased the likelihood of isolation of STEC (OR 8.5, $95 \% \mathrm{Cl} 4.4$ to 16.6$)$. 
Table 1: Clinical features of children with diarrhoea related haemolytic uraemic syndrome divided into two cohorts, 1998-2008 and 2009-2020 and entire period.

\begin{tabular}{|c|c|c|c|}
\hline & $\begin{array}{l}\text { Cohort A } \\
(1998-2008)\end{array}$ & $\begin{array}{l}\text { Cohort B } \\
(2009-2020)\end{array}$ & $\begin{array}{l}\text { Over whole period } \\
\text { n/(\%), Cohort A vs. B }\end{array}$ \\
\hline Number of children & 88 & 138 & 226 \\
\hline Mean age presentation ( \pm SEM) & $3.75 \pm 0.32$ & $3.88 \pm 0.26$ & NS \\
\hline E. coli 0157:H7 detected & 43 & 87 & $130 P=0.011$ \\
\hline \multicolumn{4}{|l|}{ Other bacteria identified } \\
\hline Campylobacter jejuni & - & 3 & \\
\hline Cryptosporidium & 1 & - & \\
\hline Aeromonas species & - & 1 & \\
\hline Viruses (Adenovirus) & - & 1 & \\
\hline Mean prodrome duration (days) & $6.9 \pm 0.4$ & $5.9 \pm 0.2$ & $6.4 p=0.04$ \\
\hline Bloody diarrhoea & 58 & 110 & $168(74.3)$ p 0.028 \\
\hline Seizures & 7 & 24 & $31(13.7 \%)$ NS \\
\hline Jaundice & 13 & 21 & $34(15) \mathrm{NS}$ \\
\hline Acute hypertension & 31 & 40 & $71(31.4)$ NS \\
\hline Anuria & 46 & 62 & $108(47.7) \mathrm{NS}$ \\
\hline Mean anuria duration (days) & $6.3 \pm 0.6$ & $5.9 \pm 0.6$ & 6.1 NS \\
\hline Number dialysed & 54 & 74 & $128(56.6) \mathrm{NS}$ \\
\hline Mean duration of dialysis & $9.4 \pm 0.9$ & $11.0 \pm 0.77$ & $10.2 \mathrm{NS}$ \\
\hline
\end{tabular}

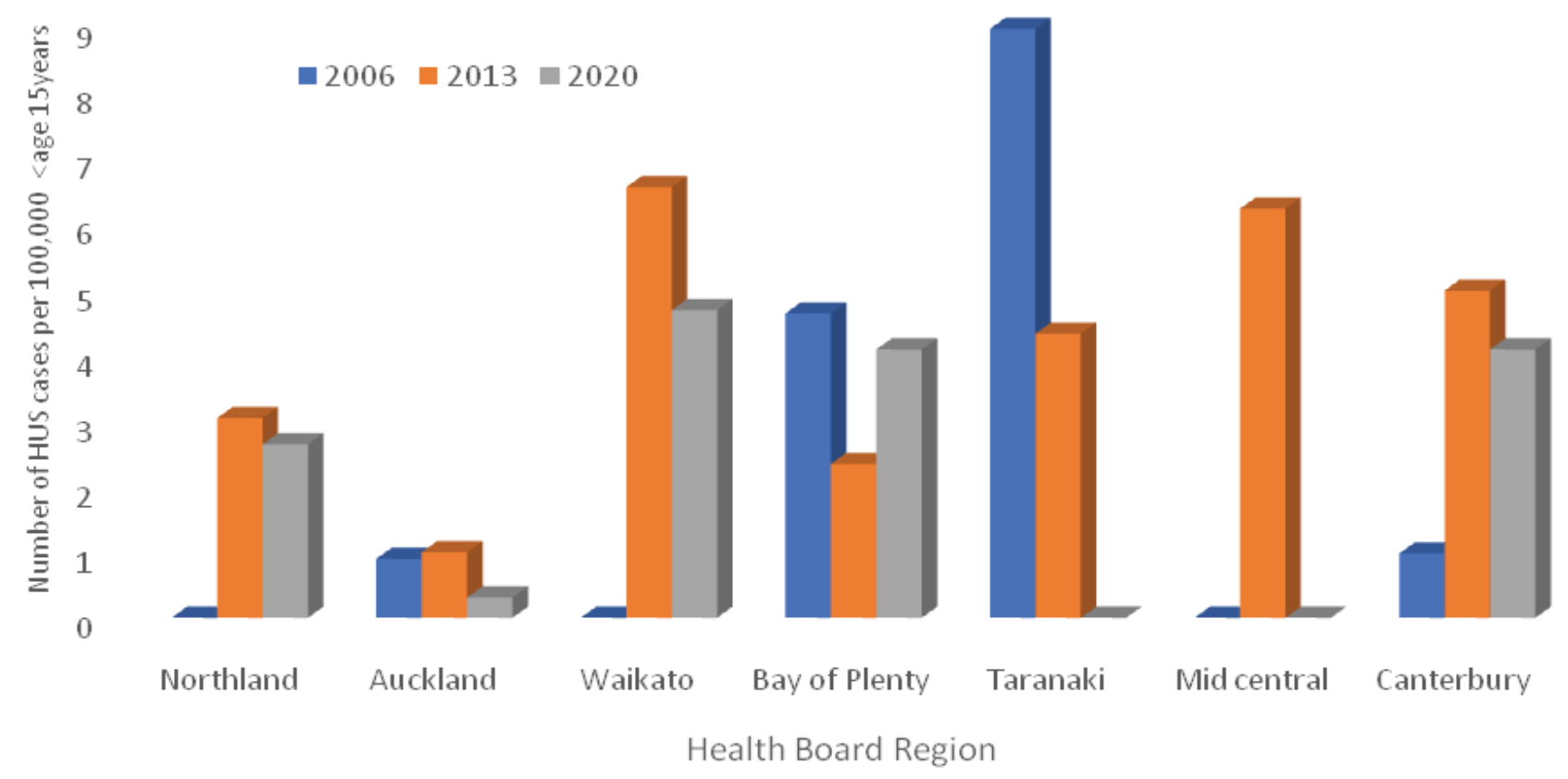

Figure 4: Children with haemolytic uraemic syndrome in seven health districts in three different years.

\section{Trends over the past 2 decades}

The incidence of children under 5 years of age developing STEC infections has gradually increased over the past two decades with a greater increase since 2012 which has been related to changes in methods of detection (Figure 2). The incidence of paediatric HUS cases over the past 23 years has fluctuated from 0.4 to 4.4 per 100,000 children with highest rates in the under five year olds, 2.85 per 100,000 from 2009 to 2020 (Figure 3), however, comparison between the two time cohorts did not show a statistically significant increase in incidence. Non E coli 0157:H7 serotypes have become more frequently isolated in the past ten years.

\section{Clinical features}

Pallor and bloody diarrhoea were common presenting symptoms (Table 1). Four patients had no history of diarrhoea but had E. coli $0157 \mathrm{H} 7$ isolated from stool specimens. Seizures occurred in $31(13.7 \%)$ children with most having one or two brief ones but six children 
displayed multiple seizures with three developing early onset and repeated generalised seizures and coma; all showed diffuse cortical and subcortical neuronal injury which resulted in a fatal outcome in three and a fourth child was successfully treated with a course of Eculizumab. The overall mortality rate for the entire period was $3 / 226$ (1.3\%). One patient had progressed to end stage renal failure after more than 10 years of chronic kidney disease and has received a successful deceased donor kidney transplant. Fifty six percent $(128 / 226)$ of the cohort required acute dialysis for acute kidney injury for a median of 9 days (range 1-35). There was no significant difference in dialysis rates between STEC positive and STEC negative children or between cohorts $A$ and $B$. All children who required acute dialysis during acute phase of the illness recovered sufficient renal function to become independent of dialysis.

\section{Discussion}

Over the two decades of this study, trends of childhood STEC infections and HUS have been observed with increasing STEC infections from both 0157: $\mathrm{H7}$ and non 0157 serotypes (Figure 5), although it has only been since 2008 that $E$. coli non 0157:H7 and non-typeable strains have been identified on a regular basis due to improved detection methods.

The Canterbury and Waikato regions have shown a trend towards increasing number of HUS cases in the second period (2009-2020) of the study. The first child with STEC HUS from the South Island was reported in 2005, eight years after the surveillance study was commenced. Prior to this time, STEC HUS in the South Island was a rarely reported, and from 2010, case numbers have steadily increased. This increase may because of the intensification of farming in these two regions. In 2019-20 report from Dairy NZ, 71.4\% of the dairy herds are in the North Island and $42 \%$ of dairy cows are in the South Island. In North Island, the Waikato region has the largest number of dairy cows $(28.5 \%)$ followed by the Taranaki region (9.6\%) In the South Island, the Canterbury region accounts almost $50 \%$ of the all dairy herds [23]. Dairy farming in the South Island has seen the most rapid growth in the past 10 years, representing $43 \%$ of total milk production in New Zealand. The increase in the childhood population in the Canterbury region was only 5\% during the study period, but the number of children with STEC-HUS had increased by $70 \%$, suggesting a possible link between an increase in dairy and cattle farming activity and STEC infections. Of the 1.187 million people who live in the South Island (up to June 2020), just over one half of the people in the South Island live in the Canterbury region. The greatest risk of STEC infection in New Zealand comes from contact with the farm environment, animal contact, untreated water supplies.

The increase in reported STEC infections over the 20 year period can only be partially explained by improved diagnostic laboratory techniques such

Annual incidence of $E$ coli 0157:H7 and non 0157 infections in children, 1998-2020

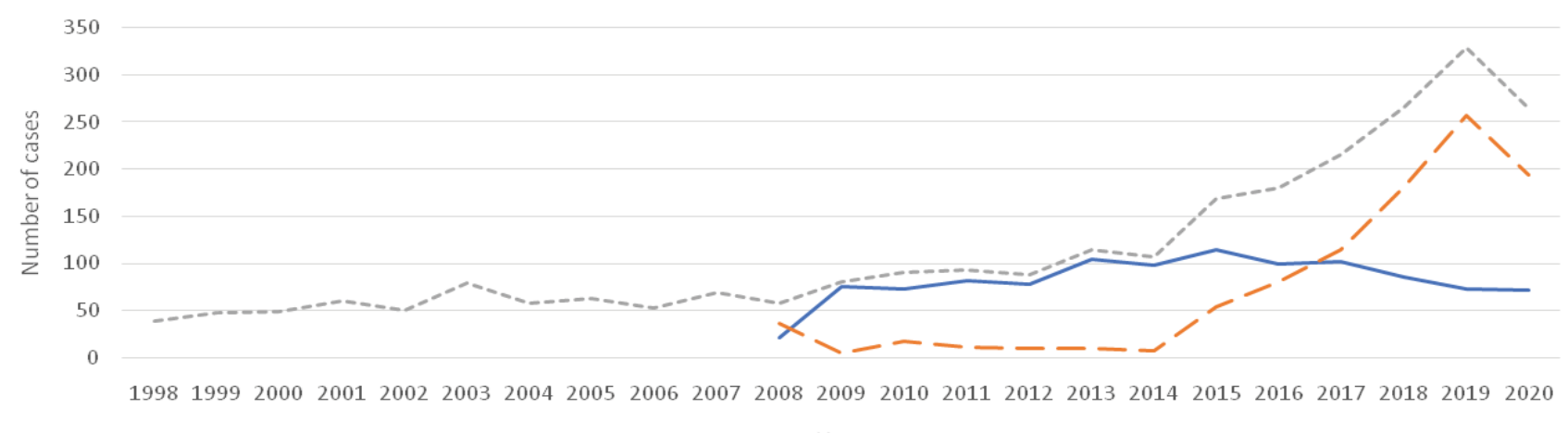

Figures 5: Annual incidence of E. coli 0157: $\mathrm{H} 7$ and non 0157 infections 1998-2020.

Figure shows the number of children under age 15 with STEC infections from 1998 to 2020. Prior to 2008, STEC isolates were classified as E. coli

O157:H7 or non typeable, but from 2008, non 0157:H7 serotypes were being identified.

Source: Institute of Environmental and Scientific Research New Zealand- https://surv.esr.cri.nz/episurv/index.php (with permission). 
as the introduction of more sensitive identification methods that utilised molecular techniques. As Figure 2 shows, the number of infections in children had been increasing from 2008, and the introduction of molecular based diagnostic assays from 2015 onwards resulted in a further rise in STEC infections isolated. (https://www.sciencelearn.org.nz/resources/2095new-zealand-dairy-farming-timeline). New Zealand has relatively high numbers of STEC cases, and contact with cattle faeces and living near cattle are risk factors for human infection. A study in 2018 assessed the national prevalence of STEC in young dairy cattle by randomly selecting 102 farms throughout New Zealand. The study used a molecular laboratory method showed STEC was found in $20 \%$ of calves on $75 \%$ of the farms studied, indicating widespread prevalence across the country [24].

During the study period, an increase in non 0157:H7 infections amongst children have also been observed. Our dataset is limited in that prior to 2008, these serotypes were not routinely and accurately identified, however, since 2008, the number of non 0157:H7 infections in children has progressively increased and has equalled 0157:H7 infections (Figure 5), but the number of non 0157:H7 associated HUS cases in children have remained very small, with only 8 children developing HUS being associated with $026(n=6), 0179(n=1)$, and $0113(n=1)$ serotypes. Shiga toxin 2 was identified in all of these cases, but eae gene was found only in the 026 isolates. The accuracy of this dataset has been verified by cross checking with information obtained from the national reference laboratory [personal communication, Institute of Environmental Scientific Research].

The association between the consumption of unpasteurised cows' milk and STEC infections in 5 young children over the study period have been sporadic and requires further monitoring. A 2014 Risk Profile of $E$. coli in Raw Milk (ESR Client Report FW13026) considered that the risk of STEC infection from the consumption of raw milk to be high [25]. Raw milk has been tested assessed for virulence gene frequencies of various serotypes of $E$. coli including 0157 , and 0103 and have been found to express the genes, but a low frequency of stx2 gene [26]. Even very low concentrations of $E$. coli 0157 in milk may represent a significant risk of infection. Similar conclusions have been obtained from both the United Kingdom and Australia [27].

Although there has been small but significant improvement in time to diagnosis between the first eleven and second twelve years of the study, some health practitioners are still not recognising the significance of a bloody diarrhoea prodrome and its association with HUS with delays in requesting appropriate stool microbiology in young children who have history of bloody diarrhoea. This has important implications as delay in stool culture from the onset of diarrhoea has an inverse effect on the return of positive stool culture for E. coli 0157:H7 [6,9] and reduces to possibility of early intervention to reduce subsequent clinical complications $[3,4]$. With the advent of rapid molecular testing being widely available in New Zealand since 2015 and the fact that STEC infections have a mandatory public health reporting pathway, delays in diagnosis should be uncommon. The general medical community remain inadequately informed of the risks of developing HUS following an enteric infection with STEC organisms. This was highlighted in a recent study which showed that emergency physicians responded in a different manner to children diagnosed with a STEC infection when compared with paediatric nephrologists [28]. Improved education of primary and secondary healthcare workers may help in early recognition and the initiation of appropriate treatment.

Other bacterial organisms that commonly cause acute bloody diarrhoea are Campylobacter, Shigella, Salmonella, Yersinia and STEC, however, of the five listed STEC is the most important due to its ability to cause HUS [29]. Shigella species causing human infection in New Zealand are not associated with HUS. Patients with STEC related bloody diarrhoea typically start with non-bloody diarrhoea initially that becomes bloody after 1-3 days. The patient is usually afebrile when they seek medical attention, and frequently have a tender abdomen [2931]. In the current study, a history of bloody diarrhoea increased the odds of an STEC infection by more than eight-fold. As soon as bloody diarrhoea is present, primary healthcare practitioners should request a stool for rapid molecular diagnostics, a full blood count and record the symptom on the request form.

Campylobacter gastroenteritis which can also cause bloody diarrhoea is highly prevalent in our community and it may therefore be difficult to distinguish an enteric infection caused by Campylobacter or STEC [32,33]. Campylobacter jejuni was the only identified bacterial organism in 3 patients with HUS. There is debate as to whether Campylobacter can cause HUS as the organism does not produce Shiga-toxin. It has been reported that up to one third of STEC gastro-intestinal infections are co- infected with other bacterial or parasitic organism [33]. We did not consider that Campylobacter infection was the cause of the HUS as their clinical course did not appear any different to STEC-HUS.

The mean incidence of STEC-HUS in New Zealand in children under age 5 years appear is higher than many other developed countries, with it increasing from 2.2 to 2.85 per 100,000 over the two time periods. In a study from Belgium between 2009 and 2015, the annual incidence in children under 5 was 1.2 per 100,000 (range 0.6-2.1) [34,35]. A study from our nearest neighbour, Australia, (1994 to 1998) reported an incidence of 1.35 per 100,000/year, but a more recent study from the same country (2000 to 2010), showed the annual 
incidence had fallen to 0.49 per 100,000 in children under age 5 [36,37]. An explanation for the difference between Australia and New Zealand may be attributed to increased environmental exposure due to the intensification of dairy farming in some areas of rural New Zealand and changes in methods of detection over time.

Seven percent $(16 / 226)$ of the HUS children in our study were under the age 12 months' of age. Infection with STEC in early infancy is thought to be rare. Our bacteriological data shows that that there has been significant increase in the cases of STEC infections over the period 2010 to 2020 , with the largest increase in the under 12-months-old group (5 fold increase in the less than 12-month-olds compared with a 2.3 fold increase in the 1-4 age group (Supplementary Data). There are few reports of STEC-HUS incidence in infants under one year of age. In the Northern Italian HUS network study, the annual incidence in children less than 12 months of age was approximately 1.2 per 100,000 aged related populations [38]. Younger children with STEC-HUS are also thought to be a higher risk of a poor outcome. A population-based survey of 10 states in the United States conducted between 1997-2012, reported 168 infants under age 2 years and calculated that this age group had a $5.4 \%$ frequency of death compared with $2.8 \%$ in the 2-4-year age group [39]. In the current study, all infants less than 12 months of age at presentation with HUS survived with just over $50 \%$ needing short term dialysis. Our small numbers of very young children in the cohort makes meaningful comparisons difficult.

There are some limitations to the results observed in this study. As with many observational studies, a large number of observers were involved in collecting clinical data from affected patients, especially in the $25 \%$ that were managed in their local hospital facilities. This variability may have introduced some inconsistencies in the interpretation of clinical symptoms, and the duration of prodromal symptoms. One of main strengths of this study is that all the patients who were transferred to our centre for clinical care were reviewed either by the author or his immediate paediatric nephrology colleagues. The other major strength of this study is the clinical and laboratory data collected from each patient remained consistent throughout the 23-year period of the study thereby allowing for trends to be recognised more readily.

\section{Conclusion}

Shiga toxin E. coli infections resulting in HUS is a common cause of acute kidney injury in young children in New Zealand requiring acute dialysis. Trends documented in this report require further study as the prevalence of STEC infections in young children continue to rise each year, with the incidence of HUS in children less than age five appears to be increasing. The consequences of a STEC infection progressing to HUS can lead to devastating consequences with permanent physical and psychological disabilities and in some rare cases, death due to severe neurological injury. Although the short-term outcome appears to be excellent, the $56 \%$ of the group who required acute renal replacement therapy are at significant risk of chronic kidney disease. Public health authorities along with other stakeholders such as the veterinary community and managers of livestock could work to identify factors to reduce the increasing exposure to the risk of STEC infections in young children.

\section{Acknowledgements}

The author wishes to thank all paediatricians who have reported children with haemolytic uraemic syndrome to the New Zealand Paediatric surveillance, University of Otago, Dunedin, New Zealand. The author also wishes to thank the scientific staff at the Institute of Environmental Science and Research of New Zealand for supplying annual data on $E$. coli serotypes, Shiga toxin gene results for the manuscript. The authors thank the Dr. Jackie Wright, Senior Scientist, Institute of Environmental Science and Research and Dr Max Morris for their input into the manuscript.

\section{Declarations}

The principal author of this manuscript is the only author.

\section{Ethics approval}

Obtained from the University of Otago, Research Ethics Committee, Dunedin, New Zealand, 1997.

Consent to collect and publish clinical data was obtained from caregivers of patients.

The dataset from which this manuscript is based upon is the property of the author and the University of Otago, Dunedin, New Zealand. Under confidentiality and privacy rules of New Zealand, the dataset cannot be deposited in a public repository without the express consent of the study participants.

\section{Competing interest}

None.

\section{Funding}

None.

\section{References}

1. Scheiring J, Andreoli SP, ZimmerhackI LB (2008) Treatment and outcome of Shiga-toxin-associated hemolytic uremic syndrome (HUS). Pediatr Nephrol 23: 1749-1760.

2. Jenssen GR, Vold L, Hovland E, Bangstad HJ, Nygård K, et al. (2016) Clinical features, therapeutic interventions and long-term aspects of hemolytic-uremic syndrome in Norwegian children: A nationwide retrospective study from 1999-2008. BMC Infect Dis 16: 285.

3. Ake JA, Jelacic S, Ciol MA, Watkins SL, Murray KF, et al. (2005) Relative nephroprotection during Escherichia coli 
O157:H7 infections: Association with intravenous volume expansion. Pediatrics 115: e673-e680.

4. Ardissino G, Tel F, Possenti I, Testa S, Consonni D, et al. (2016) Early volume expansion and outcomes of hemolytic uremic syndrome. Pediatrics 137.

5. Trachtman H, Austin C, Lewinski M, Stahl RA (2012) Renal and neurological involvement in typical Shiga toxinassociated HUS. Nat Rev Nephrol 8: 658-669.

6. Ameer MA, Wasey A, Salen P (2020) Escherichia Coli (E Coli $0157 \mathrm{H} 7$ ). In: StatPearls. StatPearls Publishing, Treasure Island (FL).

7. French multi-agency outbreak investigation team (2005) Outbreak of E. coli 0157:H7 infections associated with a brand of beefburgers in France. Euro Surveill 10.

8. Siegler R, Oakes R (2005) Hemolytic uremic syndrome; pathogenesis, treatment, and outcome. Curr Opin Pediatr 17: 200-204.

9. Tarr PI, Gordon CA, Chandler WL (2005) Shiga-toxinproducing Escherichia coli and hemolytic uraemic syndrome. Lancet 365: 1073-1086.

10. Church DL, Emshey D, Semeniuk H, Lloyd T, Pitout JD (2007) Evaluation of BBL CHROMagar 0157 versus sorbitol-MacConkey medium for routine detection of Escherichia coli 0157 in a centralized regional clinical microbiology laboratory. J Clin Microbiol 45: 3098-3100.

11. Navarro-Garcia F (2014) Escherichia coli O104:H4 Pathogenesis: An enteroaggregative E. coli/Shiga toxinproducing E. coli explosive cocktail of high virulence. Microbiol Spectr 2.

12. Balgradean M, Croitoru A, Leibovitz E (2019) An outbreak of hemolytic uremic syndrome in southern Romania during 2015-2016: Epidemiologic, clinical, laboratory, microbiologic, therapeutic and outcome characteristics. Pediatr Neonatol 60: 87-94.

13. Ingelbeen $B$, Bruyand $M$, Mariani-Kurkjian $P$, Le Hello $S$, Danis K, et al. (2018) Emerging Shiga-toxin-producing Escherichia coli serogroup $\mathrm{O} 80$ associated hemolytic and uremic syndrome in France, 2013-2016: Differences with other serogroups. PLoS One 13: e0207492.

14. Loos S, Ahlenstiel T, Kranz B, Staude H, Pape L, et al. (2012) An outbreak of Shiga toxin-producing Escherichia coli 0104:H4 hemolytic uremic syndrome in Germany: Presentation and short-term outcome in children. Clin Infect Dis 55: 753-759.

15. Carroll KJ, Harvey-Vince L, Jenkins C, Mohan K, Balasegaram S (2019) The epidemiology of Shiga toxinproducing Escherichia coli infections in the South East of England: November 2013-March 2017 and significance for clinical and public health. J Med Microbiol 68: 930-939.

16. Keenswijk W, Vanmassenhove J, Raes A, Dhont E, Vande Walle J (2017) Epidemiology and outcome of acute kidney injury in children, a single center study. Acta Clin Belg 72: 405-412.

17. Jenssen GR, Hovland E, Bangstad HJ, Nygård K, Vold L, et al. (2014) The incidence and aetiology of acute kidney injury in children in Norway between 1999 and 2008. Acta Paediatr 103: 1192-1197.

18. Ball EF, Kara T (2008) Epidemiology and outcome of acute kidney injury in New Zealand children. J Paediatr Child Health 44: 642-646.

19. Dow N, Dickson ND, Taylor B, Darlow B, Wong W, et al. (1999) The New Zealand paediatric surveillance unit: Establishment and first year of operation. New Zealand Public Health Report 6: 41-44.

20. Personal communication. Institute of Environmental Science and Research.
21. https://www.biomerieux-diagnostics.com

22. Graphpad Software. 2365 Northside Dr, Suite 560, San Diego, CA 92108, United States.

23. https://www.dairynz.co.nz/publications/dairy-industry/newzealand-dairy-statistics-2019-20/

24. Browne AS, Midwinter AC, Withers $H$, Cookson AL, Biggs PJ, et al. (2018) Molecular epidemiology of shiga toxinproducing Escherichia coli (STEC) on New Zealand dairy farms: Application of a culture-independent assay and whole-genome sequencing. Appl Environ Microbiol 84: e00481-18.

25. On S (2014) Risk profile Shiga toxin producing Escherichia coli in raw milk. Environmental Scientific Research Institute, Client report No. FW13026.

26. Douellou T, Delannoy S, Ganet S, Fach P, Loukiadis E, et al. (2017) Molecular characterisation of 0157:H7, 026:H11, 0103:H2 Shiga toxin producing $\mathrm{E}$ coli isolated from dairy products. Int J Food Microbiol 253: 59-65.

27. Costard S, Espejo L, Groenendaal H, Zagmutt FJ (2017) Outbreak-related disease burden associated with consumption of unpasteurized cow's milk and cheese, United States, 2009-2014. Emerg Infect Dis 23: 957-964.

28. Freedman SB, Grisaru S, Xie J, Samuel S, Dixon A, et al. (2018) Management of Shiga toxin producing Escherichia coli infected children: A multi-national, multi-specialty survey. J Paediatr Child Health 54: 390-397.

29. Holtz LR, Neill MA, Tarr PI (2009) Acute bloody diarrhea: A medical emergency for patients of all ages. Gastroenterology 136: 1887-1898.

30. Nester C, Andreoli SP (2016) Renal involvement in HUS. In: Pediatric Nephrology. ( $7^{\text {th }}$ edn), Avner ED, Harmon WE, Niaudet P, Yoshikawa N, Emma F, Goldstein SL.

31. Brandt ML, O'Regan S, Rousseau E, Yazbeck S (1990) Surgical complications of the haemolytic-uremic syndrome. J Pediatr Surg 25: 1109-1112.

32. https://www.health.govt.nz/our-work/diseases-andconditions/communicable-disease-control-manual/ campylobacteriosis

33. https://www.mpi.govt.nz/food-safety/food-safety-andsuitability-research/managing-the-risk-of-campylobacter/ campylobacter-research-reports/

34. Ardissino G, Possenti I, Salardi S, Tel F, Colombo E, et al. (2014) Co-infection in children with bloody diarrhea caused by Shiga toxin producing Escherichia coli: Data of the North Italian HUS network. J Pediatr Gastroenterol Nutr 59: 218220.

35. Jacquinet S, Rauw KD, Pierard D, Godefroid N, Collard L, et al. (2018) Haemolytic uremic syndrome surveillance in children less than 15 years in Belgium, 2009-2015. Archives of Public Health 76: 41.

36. Elliott EJ, Robins-Browne RM, O'Loughlin EV, BennettWood V, Bourke J, et al. (2001) Nationwide study of haemolytic uraemic syndrome: Clinical, microbiological, and epidemiological features. Arch Dis Child 85: 125-131.

37. Vally H, Hall G, Dyda A, Raupach J, Knope K, et al. (2012) Epidemiology of Shiga toxin producing Escherichia coli in Australia, 2000-2010. BMC Public Health 12: 63.

38. Ardissino G, Salardi S, Colombo E, Testa S, BorsaGhiringhelli N, et al. (2016) Epidemiology of haemolytic uremic syndrome in children. Data from the North Italian HUS network. Eur J Pediatr 175: 465-473.

39. Mody RK, Gu W, Griffin PM, Jones TF, Rounds J, et al. (2015) Postdiarrheal hemolytic uremic syndrome in united states children: Clinical spectrum and predictors of inhospital death. J Pediatr 166: 1022-1029. 
NZPSU Study: Haemolytic Uraemic Syndrome (HUS) questionnaire

\section{Reporting Details}

a. Date of report

b. Paediatrician Surname

\section{Patient Details}

a. First name (first two letters only)

b. Surname (first two letters only)

c. Date of Birth (day, month, year)

d. Age at diagnosis of HUS (yrs/months)

e. Date of diagnosis

f. Sex

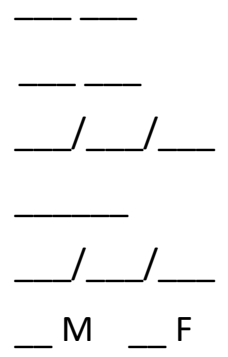

3. Historical Data (please circle one $-\mathrm{Y}=$ yes, $\mathrm{N}=$ no, $D K=$ don't know)

a. Diarrhoea prodrome

Y N DK

b. If yes, time of onset in days prior to diagnosis

days

c. If yes, did the stool contain blood

Y N DK

d. Vomiting prodrome

Y N DK

e. Respiratory prodrome

Y N DK

f. Family History of HUS

Y N DK

g. Previously/simultaneously affected sibling

Y N DK

h. Was HUS attributed to ingestion of food product?

Y N DK

i. If yes, please specify

j. Has the child visited a farm in the past 2 weeks

Y N DK

\section{Presenting or subsequent signs/symptoms (please circle)}
a. Anaemia
b. Jaundice
c. Fever
d. Oliguria
e. Anuria
f. If yes, duration
g. Hypertension
h. Seizures
i. If yes, number
j. If yes, nature eg: generalised, focal
k. Intracranial haemorrhage
I. Other (specify)

Y N DK

\begin{tabular}{lll}
$Y$ & $N$ & DK \\
$Y$ & $N$ & DK \\
$Y$ & $N$ & DK \\
$Y$ & $N$ & DK \\
$Y$ & $N$ & DK \\
& & days \\
\hline$Y$ & $N$ & DK \\
$Y$ & $N$ & $D K$ \\
\hline$Y$ & $N$ & DK \\
\hline & & \\
\hline
\end{tabular}




\section{Laboratory Findings}

a. Minimum Haemoglobin $\mathrm{g} / \mathrm{dL}$

b. Minimum Platelets $\times 10^{9} / \mathrm{L}$

c. Minimum Serum sodium $\mathrm{mmol} / \mathrm{L}$

d. Maximum Creatinine $\mu \mathrm{mol} / \mathrm{L}$

e. Maximum Urea $\mathrm{mmol} / \mathrm{L}$

f. Neutrophil count at presentation $\times 10^{9} / \mathrm{L}$

g. Abnormal RBC morphology (including fragmented or burr cells, spherocytes, polychromasia)

$$
\begin{array}{lll}
Y & N & D K \\
Y & N & D K
\end{array}
$$

h. Stool pathogen isolated

i. If yes, specify

j. Verotoxin-producing E. coli (serotype 0157:H7) isolated

Y N DK

k. Has a stool sample been sent to ESR?

$Y \quad N \quad D K$

\section{Treatment}

a. Dialysis Y N DK

b. If yes, specify type

c. Duration days

d. Other/specify

eg: supportive (hypertensives, anticonvulsants)

eg: therapeutic (fresh frozen plasma, plasmapheresis)

e. Duration of hospital care (days) days

7. Complications/Outcome (please circle)
a. Cardiomyopathy
b. Diabetes mellitus
c. Persistent hypertension
d. Persistent renal impairment
e. End-stage renal failure
f. Pancreatitis
g. Death

Y N DK

Y N DK

$Y \quad N \quad D K$

$Y \quad N \quad D K$

Y N DK

$Y \quad N \quad D K$

$Y \quad N \quad D K$

Thank you for completing this questionnaire. A short follow-up questionnaire will be sent in 12 months seeking information on long-term outcome.

\section{Please retain a copy of this form for records}

Please return to: Dr William Wong

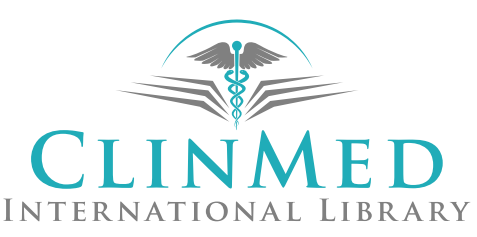

